ESAIM: PROCEEDINGS AND SURVEYS, November 2014, Vol. 46, p. 217-232

ECIT 2012 - Witold Jarczyk, Daniele Fournier-Prunaret, João Manuel Gonçalves Cabral

\title{
CURVE SHORTENING BY SHORT RULERS
}

\author{
PEter StadLeR ${ }^{1}$
}

\begin{abstract}
The aim is to construct a straight line between the two endpoints of a rectifiable curve using only a ruler, which is too short to connect them directly.

The short ruler allows it to construct a polygonal line along the curve. Consecutive midpoints of it can be connected by the short ruler. That produces a shorter polygonal line and if the shortening is iterated, the polygonal line converges to the straight line.

This short ruler method can be transported to cylinders. There, the ruler establishes short geodesics instead of straight lines. The produced sequence of curves converges to a geodesic which is homotopic to the original curve, but doesn't have to be the shortest connection between the endpoints.

Keywords. curve shortening; short ruler method; iteration; geodesic; cylinder.

Abstract in French. L'objectif est de construire une ligne droite entre les points d'extrémité d'une courbe rectifiable en utilisant seulement une règle, qui est trop court pour les connecter directement.

La règle courte permet de construire une ligne polygonale au long de la courbe. Les points centraux consécutifs de celle-ci peuvent être reliés par la règle courte. Cela produit une ligne polygonale plus courte. Si le raccourcissement est itéré, la ligne polygonale converge vers une ligne droite.

Cette méthode de la règle courte peut être généralisée à des cylindres. Dans ce cas, la règle courte établit des géodésiques courtes. La séquence de courbes converge vers une géodésique qui est homotope à la courbe initiale, mais ne doit pas nécessairement être la plus courte entre les points d'extrémité. Mots-clefs. raccourcissement des courbes; méthode de règle courte; itération; géodésique; cylindre. AMS (2000) subject classification. 26A18, 39B12, 53C22.
\end{abstract}

\section{INTRODUCTION}

We can divide the curve into sections, who are at most as long as the short ruler. It let us connect the end points of these sections creating a polygonal line with the same start and end point as the curve. We can connect consecutive midpoints of the polygonal line by the ruler and shorten it in this way.

The iteration of that midpoint method is called the short ruler method and corresponds to a difference equation. In a normed vector space the difference equation is linear and explicit solvable. The solution converges to the shortest path between start and end point: the straight line. That's the main result in the first section.

1 Technikerstraße 13, A-6020 Innsbruck

(C) EDP Sciences, SMAI 2014 

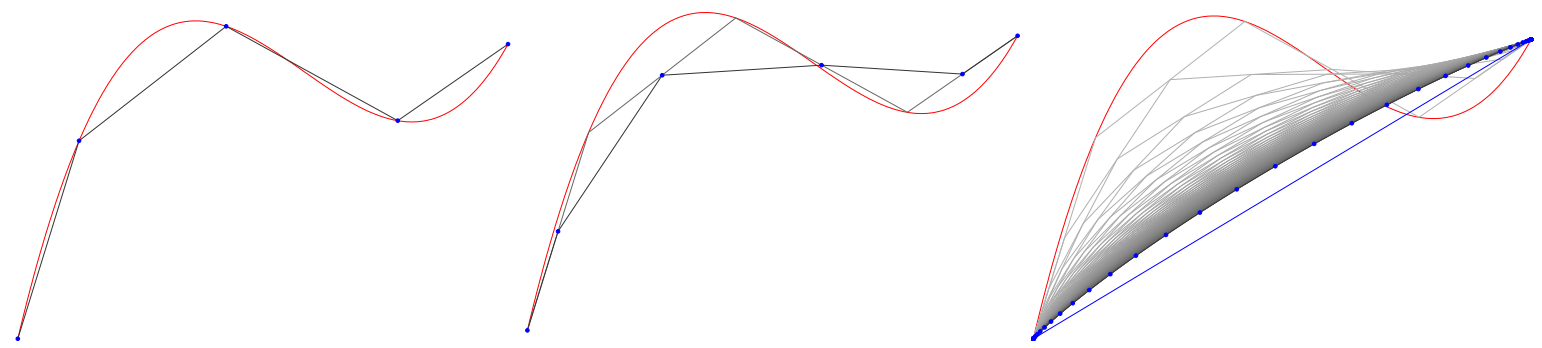

In the second section we look at cylinders, which are generated by extruding a simple closed curve. But thus, they can be unwinded into a plane and the canonical cover transports the short ruler method between plane and cylinder.

The results from the plane apply to the cylinder: Iterating the midpoint method, a curve converges to a homotopic geodesic. In contrast to straight lines in a normed vector space this geodesic doesn't have to be a shortest path on the cylinder.

\section{Normed VECTOR SPACES}

In the following section $(V,\|\cdot\|)$ denotes a normed vector space. We want to shorten an arbitrary curve in it, which is parameterized by arc length: $\alpha:[0, a L] \rightarrow V$.

After constructing a polygonal line along the path, we define the midpoint method for it. The polygonal line converges to the straight line between start and end point of $\alpha$ by iterating the midpoint method.

1.1. Remark (construction). From the path $\alpha$, we construct the nodes of a polygonal line by:

$$
p_{k}:=\alpha(k L) \text { for } k \in \mathbb{Z}
$$

Hereby, $\alpha$ is assumed to be extended to $\mathbb{R}$ constantly: $\alpha(<0)=\alpha(0)$ and $\alpha(>a L)=\alpha(a L)$. Hence, we get finitely many different points:

$$
p_{0}, \ldots p_{n} \text { with } n=\lceil a\rceil
$$

Since $\alpha$ is parameterized by arc length, the points are interconnectable by the short ruler:

$$
d\left(p_{k}, p_{k+1}\right)=d(\alpha(k L), \alpha((k+1) L)) \leq \ell\left(\left.\alpha\right|_{[k L,(k+1) L]}\right) \leq L
$$

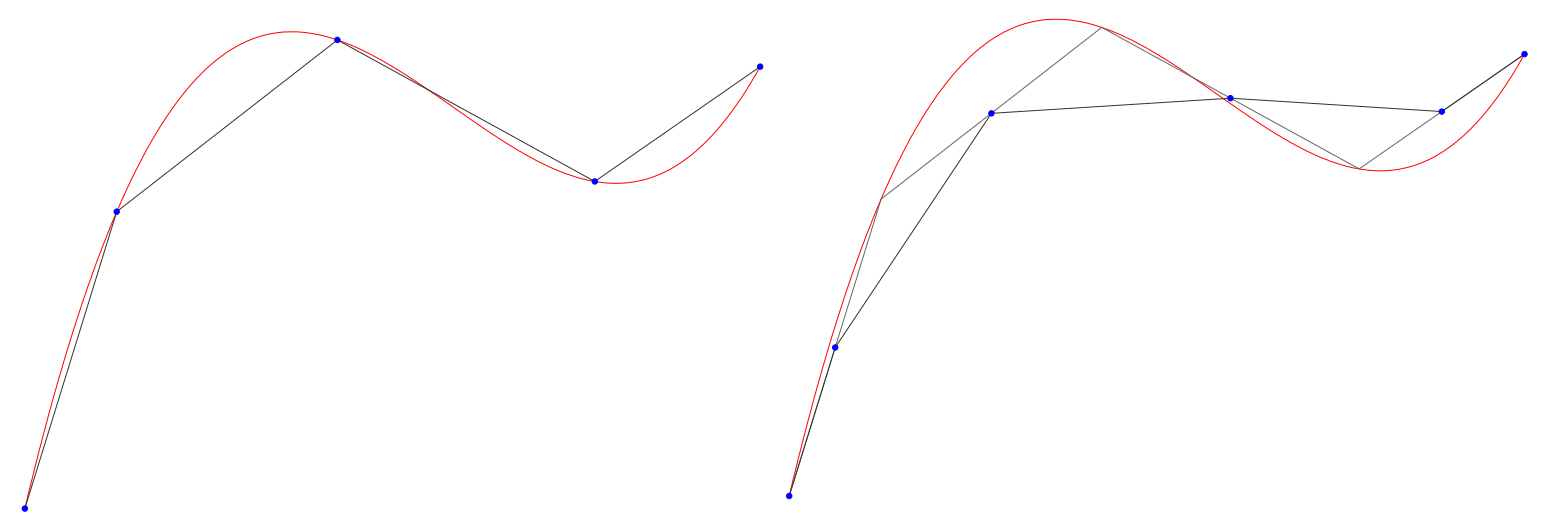

\subsection{Midpoint method}

1.2. Definition (midpoint method). Following linear operator substitutes the nodes of the polygonal line by their midpoints:

$$
S: V^{\mathbb{Z}} \longrightarrow V^{\mathbb{Z}} \text { with }(S p)_{k}=\frac{1}{2} p_{k}+\frac{1}{2} p_{k-1}
$$


It's possible to transform the constructed polygonal line in this way with the short ruler (triangle inequality).

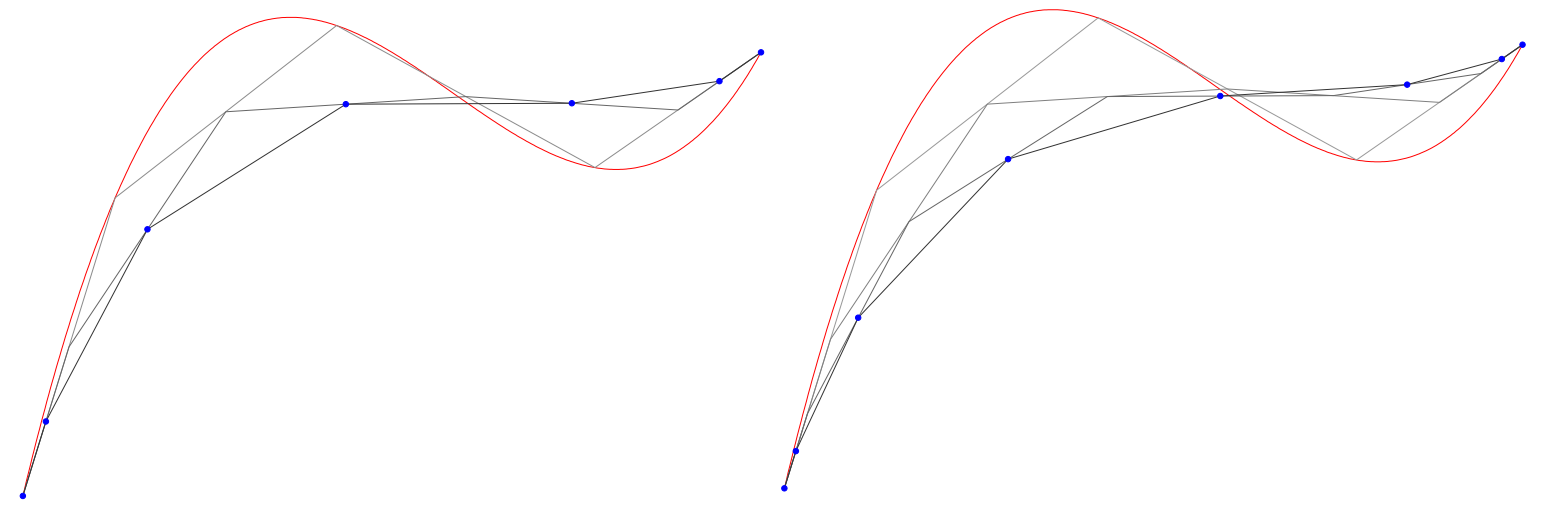

1.3. Lemma (solution). The iteration of the midpoint method is $p_{k}^{t}:=\left(S^{t}(p)\right)_{k}$ and corresponds to the partial linear difference equation

$$
p_{k}^{t+1}=\frac{1}{2}\left(p_{k}^{t}+p_{k-1}^{t}\right) \text { with initial value } p_{k}^{0}=p_{k} .
$$

This difference equation has following solution:

$$
\left(S^{t} p\right)_{k}=p_{k}^{t}=\frac{1}{2^{t}} \sum_{l=0}^{t}\left(\begin{array}{l}
t \\
l
\end{array}\right) p_{k-l}
$$

Proof by induction over $t . t=0: p_{k}^{0}=1\left(\begin{array}{l}0 \\ 0\end{array}\right) p_{k}=p_{k}$.

$$
\begin{aligned}
& t \rightarrow t+1: p_{k}^{t+1}=\left(S^{t+1} p\right)_{k}=\left(S\left(S^{t} p\right)\right)_{k}=\frac{1}{2}\left(S^{t} p\right)_{k}+\frac{1}{2}\left(S^{t} p\right)_{k-1}=\frac{1}{2} p_{k}^{t}+\frac{1}{2} p_{k-1}^{t}= \\
& =\frac{1}{2} \frac{1}{2^{t}} \sum_{l=0}^{t}\left(\begin{array}{l}
t \\
l
\end{array}\right) p_{k-l}+\frac{1}{2} \frac{1}{2^{t}} \sum_{l=0}^{t}\left(\begin{array}{l}
t \\
l
\end{array}\right) p_{k-1-l}=\frac{1}{2^{t+1}} \sum_{l=0}^{t}\left(\begin{array}{c}
t \\
l
\end{array}\right) p_{k-l}+\frac{1}{2^{t+1}} \sum_{l=1}^{t+1}\left(\begin{array}{c}
t \\
l-1
\end{array}\right) p_{k-l}= \\
& =\frac{1}{2^{t+1}}\left(p_{k}+\sum_{l=1}^{t}\left(\left(\begin{array}{c}
t \\
l
\end{array}\right)+\left(\begin{array}{c}
t \\
l-1
\end{array}\right)\right) p_{k-l}+p_{k-t-1}\right)=\frac{1}{2^{t+1}}\left(p_{k}+\sum_{l=1}^{t}\left(\begin{array}{c}
t+1 \\
l
\end{array}\right) p_{k-l}+p_{k-t-1}\right)= \\
& =\frac{1}{2^{t+1}} \sum_{l=0}^{t+1}\left(\begin{array}{c}
t+1 \\
l
\end{array}\right) p_{k-l}
\end{aligned}
$$

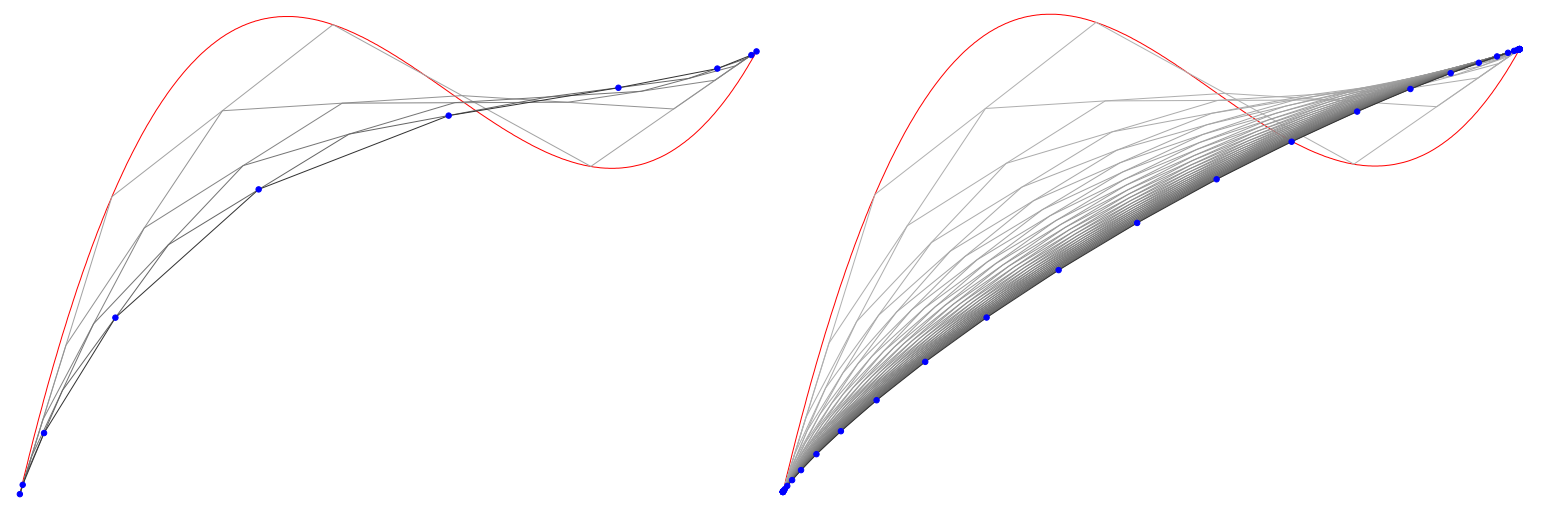




\subsection{Convergence}

1.4. Lemma. For each $t \in \mathbb{N}$ the straight line between start point $p_{0}$ and end point $p_{n}$ of the path $\alpha$ can be considered as polygonal line with nodes:

$$
g_{k}^{t}:=p_{0}+\frac{1}{2^{t}} \sum_{l=0}^{k-1}\left(\begin{array}{l}
t \\
l
\end{array}\right)\left(p_{n}-p_{0}\right) \text { with } k \in \mathbb{Z}
$$

Proof. For $t \in \mathbb{N}$ and $k \in \mathbb{Z}$ we define:

$$
a_{k}^{t}:=\frac{1}{2^{t}} \sum_{l=0}^{k-1}\left(\begin{array}{l}
t \\
l
\end{array}\right)
$$

The sequence $\left(a_{k}^{t}\right)_{k \in \mathbb{Z}}$ is monotonic increasing since no summand is negative. All $a_{k}^{t}$ are in the interval $[0,1]$, because for $k \leq 0$ it holds that $a_{k}^{t}=0$ and for $k>t$ we get with the binomial theorem:

$$
a_{k}^{t}=\frac{1}{2^{t}} \sum_{l=0}^{k-1}\left(\begin{array}{l}
t \\
l
\end{array}\right)=\frac{1}{2^{t}} \sum_{l=0}^{t}\left(\begin{array}{l}
t \\
l
\end{array}\right)+0=\frac{1}{2^{t}}(1+1)^{t}=1
$$

For $k \in \mathbb{Z}$ the points $g_{k}^{t}=p_{0}+a_{k}^{t}\left(p_{n}-p_{0}\right)$ are in a row on the straight line between start and end point:

$$
g_{\leq 0}^{t}=p_{0}+a_{\leq 0}^{t}\left(p_{n}-p_{0}\right)=p_{0} \text { and } g_{>t}^{t}=p_{0}+a_{>t}^{t}\left(p_{n}-p_{0}\right)=p_{n}
$$

The polygonal line with nodes $\left(g_{k}^{t}\right)_{k \in \mathbb{Z}}$ represents the straight line between $p_{0}$ and $p_{n}$.

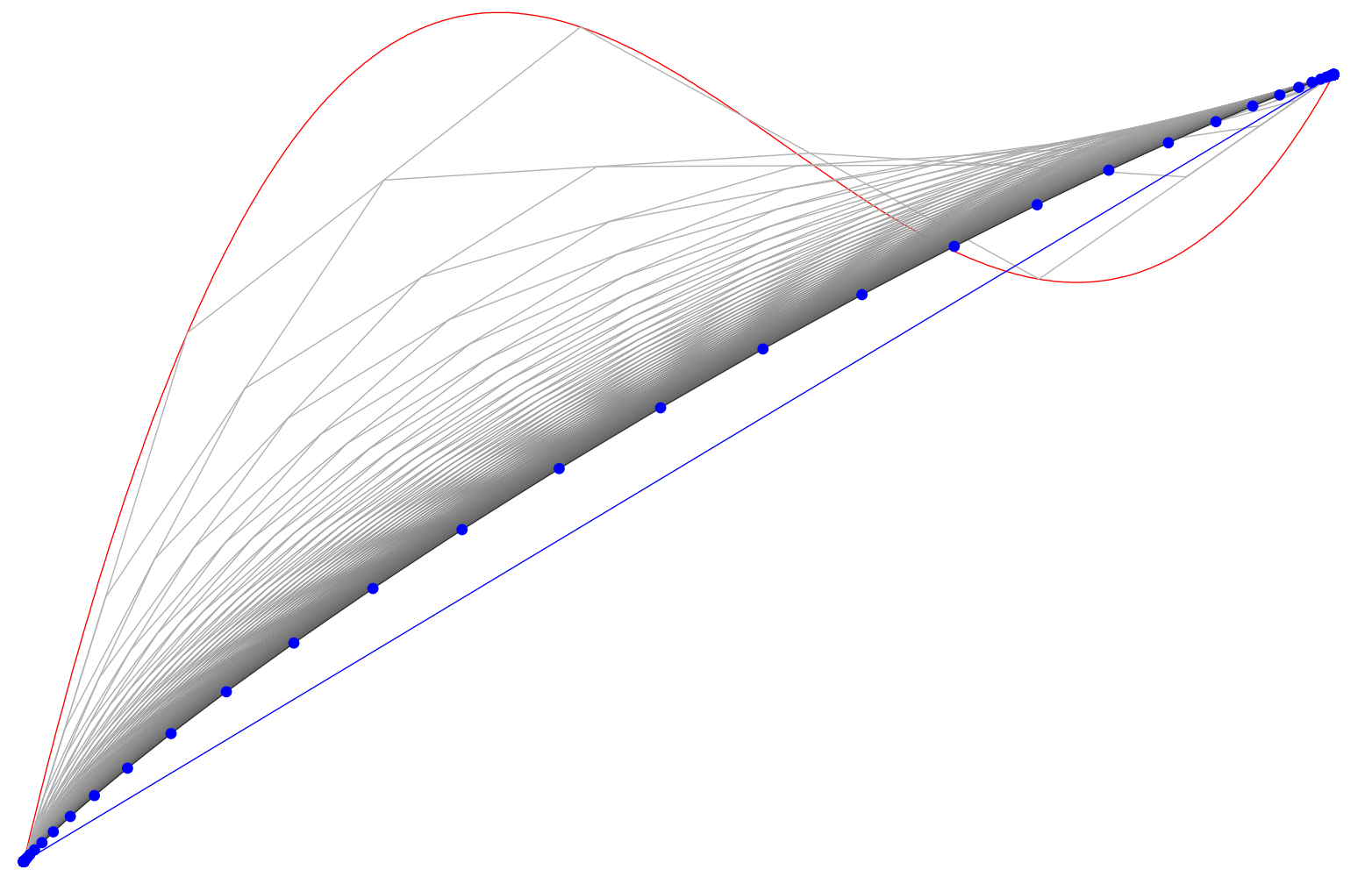


1.5. Theorem (result). The polygonal lines converge uniformly in the normed vector space $V$ to the straight line between start and end point of the path $\alpha$ :

$$
\forall \varepsilon>0: \exists r \in \mathbb{N}: \forall t>r: \sup _{k \in \mathbb{Z}}\left\|\left(S^{t} p\right)_{k}-g_{k}^{t}\right\|<\varepsilon
$$

Proof. We define $g_{\leq 0}:=p_{0}$ and $g_{>0}:=p_{n}$. That's just the sequence from Lemma 1.4 for $t=0$ and at the end of the proof, we will see that transforming these nodes of the straight line by the midpoint method produces other nodes of the same straight line: $\left(S^{t} g\right)_{k}=p_{k}^{t}$.

The difference is $y_{l}:=(p-g)_{l}=0$ for $l \in \mathbb{Z} \backslash\{1, \ldots n-1\}$ and the maximum $M:=\max _{l \in \mathbb{Z}}\left\|y_{l}\right\|$ exists.

With $\left(\begin{array}{c}2 t \\ l\end{array}\right) \leq\left(\begin{array}{c}2 t \\ t\end{array}\right)$ and lemma 1.3 it holds that:

$$
\begin{aligned}
\left\|\left(S^{2 t} y\right)_{k}\right\| & =\left\|\frac{1}{4^{t}} \sum_{l=0}^{2 t}\left(\begin{array}{c}
2 t \\
l
\end{array}\right) y_{k-l}\right\| \leq \frac{1}{4^{t}} \sum_{l=0}^{2 t}\left(\begin{array}{c}
2 t \\
l
\end{array}\right)\left\|y_{k-l}\right\| \leq \frac{1}{4^{t}} \sum_{l=0}^{2 t}\left(\begin{array}{c}
2 t \\
t
\end{array}\right)\left\|y_{k-l}\right\| \leq \frac{1}{4^{t}} \sum_{l=0}^{n}\left(\begin{array}{c}
2 t \\
t
\end{array}\right)\left\|y_{l}\right\| \leq \\
& \leq \frac{1}{4^{t}} \sum_{l=0}^{n}\left(\begin{array}{c}
2 t \\
t
\end{array}\right) \cdot M=\frac{1}{4^{t}}\left(\begin{array}{c}
2 t \\
t
\end{array}\right) \cdot M \cdot(n+1)
\end{aligned}
$$

The numbers $n$ and $M$ are constant and for $x_{t}:=\frac{1}{4^{t}}\left(\begin{array}{c}2 t \\ t\end{array}\right)=\frac{(2 t) !}{4^{t}(t !)^{2}}$ it holds that $x_{0}=1$ and for $j \in \mathbb{N}_{>0}$ :

$$
\frac{x_{j}}{x_{j-1}}=\frac{2 j(2 j-1)(2 j-2) !}{4 j^{2}((j-1) !)^{2}} \cdot \frac{4^{j-1}((j-1) !)^{2}}{(2 j-2) !}=\frac{\not \not p(2 j-1)}{\not 2 \cdot 2 j^{\not 2}}=1-\frac{1}{2 j} \geq 0
$$

Since $1+s \leq \exp (s)$ for $s \in \mathbb{R}$, the sequence $\left(x_{t}\right)_{t}$ converges to 0 :

$$
0 \leq x_{t}=\prod_{j=1}^{t} \frac{x_{j}}{x_{j-1}}=\prod_{j=1}^{t}\left(1-\frac{1}{2 j}\right) \leq \prod_{j=1}^{t} \exp \left(-\frac{1}{2 j}\right)=\exp \left(-\sum_{j=1}^{t} \frac{1}{2 j}\right) \longrightarrow 0
$$

Therefore, the subsequence $\left(S^{2 t} y\right)_{t \in \mathbb{N}}$ converges uniformly to 0 .

With $\left(\begin{array}{c}2 t-1 \\ l\end{array}\right) \leq\left(\begin{array}{c}2 t \\ l\end{array}\right) \leq\left(\begin{array}{c}2 t \\ t\end{array}\right)$ and lemma 1.3 the same result holds for the subsequence $\left(S^{2 t-1} y\right)_{t}$ :

$$
\begin{aligned}
\left\|\left(S^{2 t-1} y\right)_{k}\right\| & =\left\|\frac{2}{4^{t}} \sum_{l=0}^{2 t-1}\left(\begin{array}{c}
2 t-1 \\
l
\end{array}\right) y_{k-l}\right\| \leq \frac{2}{4^{t}} \sum_{l=0}^{2 t-1}\left(\begin{array}{c}
2 t-1 \\
l
\end{array}\right)\left\|y_{k-l}\right\| \leq \frac{2}{4^{t}} \sum_{l=0}^{2 t-1}\left(\begin{array}{c}
2 t \\
t
\end{array}\right)\left\|y_{k-l}\right\| \leq \frac{2}{4^{t}} \sum_{l=0}^{n}\left(\begin{array}{c}
2 t \\
t
\end{array}\right)\left\|y_{l}\right\| \leq \\
& \leq \frac{2}{4^{t}} \sum_{l=0}^{n}\left(\begin{array}{c}
2 t \\
t
\end{array}\right) \cdot M=\frac{2}{4^{t}}\left(\begin{array}{c}
2 t \\
t
\end{array}\right) \cdot M \cdot(n+1)=x_{t} \cdot 2 \cdot M \cdot(n+1)
\end{aligned}
$$

Therefore $\forall \varepsilon>0: \exists r \in \mathbb{N}: \forall t>r: \sup _{k \in \mathbb{Z}}\left\|\left(S^{t}(p-g)\right)_{k}\right\|<\varepsilon$. The operator $S$ is linear and $S^{t}(p-g)=S^{t} p-S^{t} g$ converges uniformly to 0 . Given lemma 1.3 and the definition of $g$, it holds that:

$$
\left(S^{t} g\right)_{k}=\frac{1}{2^{t}} \sum_{l=0}^{t}\left(\begin{array}{l}
t \\
l
\end{array}\right) g_{k-l}=\frac{1}{2^{t}} \sum_{l=0}^{k-1}\left(\begin{array}{l}
t \\
l
\end{array}\right) g_{>0}+\frac{1}{2^{t}} \sum_{l=k}^{t}\left(\begin{array}{l}
t \\
l
\end{array}\right) g_{\leq 0}=\frac{1}{2^{t}} \sum_{l=0}^{k-1}\left(\begin{array}{l}
t \\
l
\end{array}\right) p_{n}+\frac{1}{2^{t}} \sum_{l=k}^{t}\left(\begin{array}{l}
t \\
l
\end{array}\right) p_{0}=g_{k}^{t}
$$

According to lemma 1.4 , these $g_{k}^{t}$ represent the straight line between $p_{0}$ and $p_{n}$. 
Altogether, the polygonal lines with nodes $S^{t} p$ converge uniformly to this straight line between start and end point of the path $\alpha$.

\section{Cylinder}

Extruding the image of a simple closed curve generalizes a cylinder: $\mathcal{Z} \subseteq \mathbb{R}^{2} \times \mathbb{R}^{m}$. Again, we want to shorten an arbitrary curve in it, which is parameterized by arc length: $\beta:[0, a L] \rightarrow \mathcal{Z}$.

For this, we unwind the generalized cylinder along the curve into the vector space $\mathbb{R} \times \mathbb{R}^{m}$. Thereby the short ruler method for vector spaces is transported to the cylinder producing polygonal geodesics. These converge to a geodesic, which connects start and end point of the curve $\beta$.

2.1. Definition (generalized cylinder). A simple closed path $\xi:[0, \lambda] \rightarrow \mathbb{R}^{2}$, which is parameterized by arc length, generates a generalized cylinder:

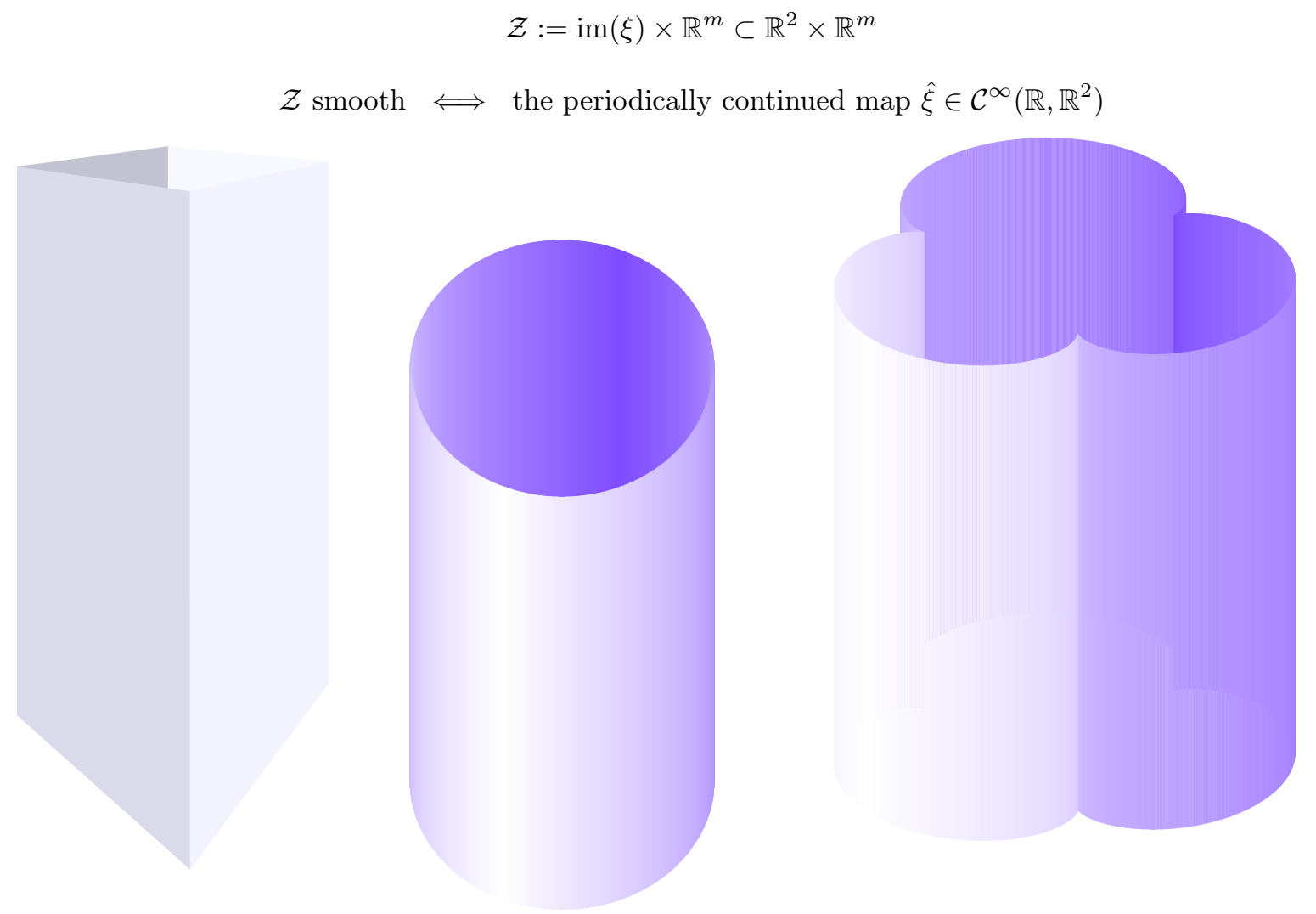

2.2. Lemma. Let $\xi:[0, \lambda] \rightarrow \mathbb{R}^{2}$ be a simple connected path and $\hat{\xi}$ its periodical extension to $\mathbb{R}$. For every $t \in \mathbb{R}$ the following restriction of $\hat{\xi}$ is homeomorphic:

$$
\left.\left.\hat{\xi}\right|_{] t, t+\lambda[}:\right] t, t+\lambda[\stackrel{\cong}{\longrightarrow} \operatorname{im}(\xi) \backslash\{\hat{\xi}(t)\}
$$

Proof. Let be $t \in \mathbb{R}$.

(a) The restriction $\left.\hat{\xi}\right|_{] t, t+\lambda[}$ is continuous since $\hat{\xi}$ is continuous.

(b) If $s \in \mathbb{R}$, then:

$$
s-\left\lfloor\frac{s}{\lambda}\right\rfloor \lambda \in\left[0, \lambda\left[\text { and } \hat{\xi}(s)=\xi\left(s-\left\lfloor\frac{s}{\lambda}\right\rfloor \lambda\right)\right.\right.
$$


For all $\sigma, \tau \in[t, t+\lambda[$ with same function value $\hat{\xi}(\tau)=\hat{\xi}(\sigma)$ it holds that:

$$
\xi\left(\tau-\left\lfloor\frac{\tau}{\lambda}\right\rfloor \lambda\right)=\hat{\xi}(\tau)=\hat{\xi}(\sigma)=\xi\left(\sigma-\left\lfloor\frac{\sigma}{\lambda}\right\rfloor \lambda\right)
$$

Thereby, all arguments of $\xi$ are in $[0, \lambda[$. But the restriction of the simple closed path $\xi$ to $[0, \lambda[$ is injective:

$$
\tau-\left\lfloor\frac{\tau}{\lambda}\right\rfloor \lambda=\sigma-\left\lfloor\frac{\sigma}{\lambda}\right\rfloor \lambda
$$

Hence, it's $\tau \in \sigma+\mathbb{Z} \lambda$. On the other side, $|\tau-\sigma|$ is less than $\lambda$ and $\tau$ equals $\sigma$.

This holds for arbitrary arguments, the restriction $\left.\hat{\xi}\right|_{[t, t+\lambda[}$ is injective.

(c) Since $\lambda$ is the period of $\hat{\xi}$, the restriction $\left.\hat{\xi}\right|_{[t, t+\lambda[}$ has the same image as $\xi$.

Together with (b) $\left.\hat{\xi}\right|_{[t, t+\lambda[}:[t, t+\lambda[\longrightarrow \operatorname{im}(\xi)$ is bijective. That doesn't change if the argument $t$ and the corresponding function value are eliminated:

$$
\left.\left.\hat{\xi}\right|_{] t, t+\lambda[}:\right] t, t+\lambda[\longleftrightarrow \operatorname{im} \xi \backslash\{\hat{\xi}(t)\}
$$

(d) The inverse of the bijection from (c) is continuous, i.e., the bijection is an open map:

$$
\forall r \in] t, t+\lambda[: \forall \varepsilon>0: \exists \delta>0: \forall s \in] t, t+\lambda[:\|\hat{\xi}(s)-\hat{\xi}(r)\|<\delta \Rightarrow|s-r|<\varepsilon
$$

That's shown indirectly: Let be $r \in] t, t+\lambda[$ and $\varepsilon>0$. Then for every $\delta>0$ there would exist an $s \in] t, t+\lambda[$ with $|s-r| \geq \varepsilon$ and:

$$
\|\hat{\xi}(s)-\hat{\xi}(r)\|<\delta
$$

Given the continuity of $\hat{\xi}$ on $\mathbb{R}$ in the closure of $] t, t+\lambda[$ there would be another argument $s \in[t, t+\lambda]$ with $\hat{\xi}(s)=\hat{\xi}(r)$ and $|s-r| \geq \varepsilon$, therefore would be $s \neq r$.

If $s=t+\lambda$, the arguments $t$ and $r$ would have the same function value $\hat{\xi}(r)=\hat{\xi}(s)=\hat{\xi}(t+\lambda)=\hat{\xi}(t)$, since $\hat{\xi}$ is periodic. But they must be different, because $r \in] t, t+\lambda[$.

Without loss of generality, there would be an argument $s \in[t, t+\lambda[$, which is different from $r$ but has the same function value. As that's a contradiction to the injectivity of $\hat{\xi}$ on $[t, t+\lambda[$ (b), the bijection has to be open.

Altogether, the restriction is homeomorphic.

2.3. Definition. A subset $A$ of a topological space $B$ is discrete iff all $a \in A$ are isolated, i.e. if there are neighborhoods $V$ in $B$, such that $A \cap V=\{a\}$.

For another topological space $X$ a continuous function $\pi: B \rightarrow X$ is called discrete iff the set $\pi^{-1}\{x\}$ is discrete for every $x \in X$. [3, p. 20]

2.4. Definition. A discrete function $\pi: B \rightarrow X$ from one topological space to another is a cover of $X$ iff for all $x \in X$ exists an open neighborhood $U$ of $x$ and a homeomorphism $\phi: \pi^{-1}(U) \longrightarrow U \times \pi^{-1}\{x\}$, such that the projection is $\operatorname{pr}_{U} \circ \phi=\left.\pi\right|_{\pi^{-1}(U)}$. [1, p. $153 \mathrm{f}$.].

2.5. Lemma. If the path $\xi:[0, \lambda] \rightarrow \mathbb{R}^{2}$ is simple closed, parameterized by arc length and has the periodic continuation $\hat{\xi}$ to $\mathbb{R}$, then $\pi:=\hat{\xi} \times \operatorname{id}_{\mathbb{R}^{m}}$ covers the generalized cylinder $\mathcal{Z}=\operatorname{im}(\xi) \times \mathbb{R}^{m}$.

Proof. (a) The function $\pi=\hat{\xi} \times$ id: $\mathbb{R} \times \mathbb{R}^{m} \rightarrow \mathcal{Z}$ is continuous since $\hat{\xi}$ and the identity on $\mathbb{R}^{m}$ are continuous.

(b) Let be $(z, v) \in \mathcal{Z} \subset \mathbb{R}^{2} \times \mathbb{R}^{m}$ arbitrary. Then $z$ is in the image of the injective function $\left.\xi\right|_{[0, \lambda[}$, has an unique preimage under it and has the following preimage under the periodic continuation $\hat{\xi}$ :

$$
\hat{\xi}^{-1}\{z\}=\left.\xi\right|_{[0, \lambda[} ^{-1}(z)+\lambda \mathbb{Z}
$$


The preimage of $(z, v)$ under $\pi$ is discrete, because it can be identified with the discrete set $\mathbb{Z}$ :

$$
\begin{gathered}
\pi^{-1}\{(z, v)\}=\left\{(\tau, u) \in \mathbb{R} \times \mathbb{R}^{m} \mid \hat{\xi}(\tau)=z \text { und } u=v\right\}= \\
=\left(\left.\xi\right|_{[0, \lambda[} ^{-1}(z)+\lambda \mathbb{Z}\right) \times\{v\} \cong \mathbb{Z}:(x, v) \longleftrightarrow \frac{x-\left.\xi\right|_{[0, \lambda[} ^{-1}(z)}{\lambda}
\end{gathered}
$$

Since every $(v, z)$ has a discrete preimage, the function $\pi$ is discrete.

(c) Let be $t \in \mathbb{R}$ with $\hat{\xi}(t) \neq z$, e.g. $t=\left.\xi\right|_{[0, \lambda[} ^{-1}(z)+\frac{\lambda}{2}$, and:

$$
U:=\mathcal{Z} \backslash\left(\{\hat{\xi}(t)\} \times \mathbb{R}^{m}\right)=(\operatorname{im}(\xi) \backslash\{\hat{\xi}(t)\}) \times \mathbb{R}^{m}
$$

Then, $U$ is an open neighborhood of $(z, v)$ and like in (b) it has the preimage:

$$
\pi^{-1}(U)=\bigcup_{(x, u) \in U}\left(\left.\xi\right|_{[0, \lambda[} ^{-1}(x)+\lambda \mathbb{Z}\right) \times\{u\}=(] t, t+\lambda[+\lambda \mathbb{Z}) \times \mathbb{R}^{m}
$$

(d) Given that the cover $\pi=\hat{\xi} \times$ id is continuous, the following function is continuous, too:

$$
\phi: \pi^{-1}(U) \longrightarrow U \times \mathbb{Z}:(\tau, u) \longmapsto\left((\hat{\xi}(\tau), u),\left\lfloor\frac{\tau-t}{\lambda}\right\rfloor\right)
$$

The identity id: $\mathbb{R}^{m} \rightarrow \mathbb{R}^{m}$ is homeomorphic and together with Lemma 2.2 we get the homeomorphism:

$$
\left.\left.\hat{\xi}\right|_{] t, t+\lambda[} ^{-1} \times \operatorname{id}: U=(\operatorname{im}(\xi) \backslash\{\hat{\xi}(t)\}) \times \mathbb{R}^{m} \longrightarrow\right] t, t+\lambda\left[\times \mathbb{R}^{m}=: V\right.
$$

The image $V$ creates the domain of $\phi$, because with (c) we can write $\pi^{-1}(U)$ as $V+\lambda \mathbb{Z} \times\{0\}$. Thus, $\phi$ has the continuous inverse:

$$
\phi^{-1}: U \times \mathbb{Z} \longrightarrow \pi^{-1}(U),((x, u), k) \longmapsto\left(k \lambda+\left.\hat{\xi}\right|_{] t, t+\lambda[} ^{-1}(x), u\right)
$$

(e) Altogether, $\phi$ is homeomorphic and $\operatorname{pr}_{U} \circ \phi=\left.(\hat{\xi} \times \mathrm{id})\right|_{\pi^{-1}(U)}=\left.\pi\right|_{\pi^{-1}(U)}$.

\subsection{Unwinding}

2.6. Definition. Let be $B$ and $X$ topological spaces with a path $\beta:[r, s] \rightarrow X$ and a continuous function $\pi: B \rightarrow X$. A path $\alpha:[r, s] \rightarrow B$ is called a lift of $\beta$ over $\pi$ to $B$ iff $\pi \circ \alpha=\beta$.

2.7. Theorem. For topological spaces $B$ and $X$ and a cover $\pi: B \rightarrow X$, every path in $X$ can be lifted uniquely over $\pi$ to $B$ after choosing a start point:

$$
\forall \beta \in \mathcal{C}^{0}([r, s], X): \forall w \in \pi^{-1}\{\beta(r)\}: \exists_{1} \alpha \in \mathcal{C}^{0}([r, s], B): \pi \circ \alpha=\beta, \alpha(r)=w
$$

Proof. (see [1, p. 161 f.] or [2, p. 124 f.])

Given the definition 2.4 of the cover, for each point $x \in X$ we denote the neighborhood of $x$ with $U_{x}$ and the corresponding homeomorphism with $\phi_{x}$ such that:

$$
\mathrm{pr}_{U_{x}} \circ \phi_{x}=\left.\pi\right|_{\pi^{-1}\left(U_{x}\right)}
$$


(a) The lift is unique: Paths in $B$ are equal if they have the same start point and are equal after applying the cover to them. Let be $\alpha$ and $\tilde{\alpha}$ paths in $B$, which have the same start point $\alpha(r)=\tilde{\alpha}(r)$ and are equal if the cover is applied:

$$
\pi \circ \alpha=\pi \circ \tilde{\alpha}
$$

We have to show, that the two paths equal on the whole domain $[r, s]$. The interval $[r, s]$ is connected and the following set is not empty:

$$
r \in M:=\{\tau \in[r, s] \mid \alpha(\tau)=\tilde{\alpha}(\tau)\} \subseteq[r, s]
$$

If $M$ is both open and closed in $[r, s]$, then $M=[r, s]$.

The definition of the map $(\tilde{\alpha}, \alpha):[r, s] \longrightarrow B \times_{X} B:=\{(u, v) \in B \times B \mid \pi(u)=\pi(v)\}$ makes sense, because both paths equal after applying the cover $\pi$. The function is continuous and maps $M$ onto the diagonal:

$$
(\tilde{\alpha}, \alpha)(M)=\{(\tilde{\alpha}(\tau), \alpha(\tau)) \mid \tau \in M\}=\{(\alpha(\tau), \alpha(\tau)) \mid \tau \in M\} \subseteq \Delta:=\{(u, u) \mid u \in B\} \subseteq B \times \times_{X} B
$$

The diagonal $\Delta$ is closed: For $(u, v) \in B \times_{X} B \backslash \Delta$ the two elements $u$ and $v$ are different, but both have the same image $y:=\pi(u)=\pi(v)$. The open neighborhoods $U:=\phi_{y}^{-1}\left(U_{y} \times\{u\}\right)$ and $V:=\phi_{y}^{-1}\left(U_{y} \times\{v\}\right)$ of $u$ and $v$, respectively, are disjoint, because $\phi_{y}$ is homeomorphic and $u \neq v$. Hence, the product $U \times V$ is disjoint to the diagonal $\Delta$ and $(u, v)$ has the following open neighborhood in $B \times_{X} B$ :

$$
(U \times V) \cap\left(B \times_{X} B\right) \subseteq\left(B \times_{X} B\right) \backslash \Delta
$$

Thus, $M$ is closed as the preimage of the diagonal $\Delta$ under the continuous function $(\tilde{\alpha}, \alpha)$. On the other side, $M$ is open: For $\tau \in M$ both paths have the same image $v:=\tilde{\alpha}(\tau)=\alpha(\tau)$ and for the open set $U_{\pi(v)} \times\{v\}$ in the image of the homeomorphism $\phi_{\pi(v)}$ there's an open subset $T \subseteq[r, s]$, such that:

$$
\phi_{\pi(v)}(\tilde{\alpha}(T)) \subseteq U_{\pi(v)} \times\{v\} \text { and } \phi_{\pi(v)}(\alpha(T)) \subseteq U_{\pi(v)} \times\{v\}
$$

Therefore and since $\pi \circ \tilde{\alpha}=\pi \circ \alpha$, for all $\sigma \in T$ the projection pr: $U_{\pi(v)} \times\{v\} \rightarrow U_{\pi(v)}$ yields:

$$
\operatorname{pr}\left(\phi_{\pi(v)}(\tilde{\alpha}(\sigma))\right)=\pi(\tilde{\alpha}(\sigma))=\pi(\alpha(\sigma))=\operatorname{pr}\left(\phi_{\pi(v)}(\alpha(\sigma))\right)
$$

This projection pr and the $\phi_{\pi(v)}$ are homeomorphic and for $\sigma \in T$ it holds that:

$$
\tilde{\alpha}(\sigma)=\alpha(\sigma)
$$

Thus, $T \subseteq M$ is a neighborhood of $\tau$. Since we can choose $\tau \in M$ arbitrarily, the set $M$ is open. Again $M$ is closed, non-empty $(r \in M)$ and a subset of a connected set and hence equal to it: $M=[r, s]$.

(b) For the existence of a lift let $\beta:[r, s] \rightarrow X$ be a path and $w \in \pi^{-1}\{\beta(r)\}$ a start point. Without loss of generality, we assume $r=0$ and $s=1$.

The open neighborhoods $\left(U_{x}\right)_{x \in X}$ cover the set $X$. The continuous function $\beta$ produces an open cover of the compact interval $[0,1]$ :

$$
\left(\beta^{-1}\left(U_{x}\right)\right)_{x \in X}
$$

There exists a Lebesgue number $1 / n$ with $n \in \mathbb{N}$ : In the open cover $\left(U_{x}\right)_{x \in X}$ there's a neighborhood for each subset of the interval $[0,1]$, whose diameter is at most $1 / n$.

For $k \in\{0, \ldots n-1\}$ the sub interval $\left[\frac{k}{n}, \frac{k+1}{n}\right]$ has diameter $1 / n$. Let be $x_{k} \in X$ the point, whose corresponding open set $\beta^{-1}\left(U_{x_{k}}\right)$ covers this interval:

$$
\left[\frac{k}{n}, \frac{k+1}{n}\right] \subset \beta^{-1}\left(U_{x_{k}}\right) \text { and } \beta\left[\frac{k}{n}, \frac{k+1}{n}\right] \subset U_{x_{k}}
$$


(c) We define $\alpha:[0,1] \rightarrow B$ recursive by setting $\alpha(0):=w \in \pi^{-1}\{\beta(0)\}$ as start point.

And if $\alpha(k / n) \in \pi^{-1}\{\beta(k / n)\}$ is defined for one $k \in\{0, \ldots n-1\}$, we set:

$$
\left.\left.\alpha(\tau):=\phi_{x_{k}}^{-1}\left(\beta(\tau), \operatorname{pr}_{\pi^{-1}\left\{x_{k}\right\}} \circ \phi_{x_{k}}\left(\alpha\left(\frac{k}{n}\right)\right)\right) \text { for } \tau \in\right] \frac{k}{n}, \frac{k+1}{n}\right]
$$

This definition makes sense, because $\phi_{x_{k}}: \pi^{-1}\left(U_{x_{k}}\right) \rightarrow U_{x_{k}} \times \pi^{-1}\left(x_{k}\right)$ is homeomorphic and with (b) is:

$$
\left.\beta] \frac{k}{n}, \frac{k+1}{n}\right] \subset U_{x_{k}} \text { and } \alpha\left(\frac{k}{n}\right) \in \pi^{-1}\left\{\beta\left(\frac{k}{n}\right)\right\} \subset \pi^{-1}\left(U_{x_{k}}\right)
$$

Moreover, $\alpha\left(\frac{k+1}{n}\right)$ is in the preimage $\pi^{-1}\left\{\beta\left(\frac{k+1}{n}\right)\right\}$, because for all $\left.\left.\tau \in\right] \frac{k}{n}, \frac{k+1}{n}\right]$ it holds that:

$$
\pi \circ \alpha(\tau)=\pi \circ \phi_{x_{k}}^{-1}\left(\beta(\tau), \operatorname{pr}_{\pi^{-1}\left\{x_{k}\right\}} \circ \phi_{x_{k}}\left(\alpha\left(\frac{k}{n}\right)\right)\right)=\operatorname{pr}_{U_{x_{k}}}(\beta(\tau), \ldots)=\beta(\tau)
$$

Thus, $\alpha$ is well-defined on the whole interval $[0,1]$.

(d) On the sub intervals $\alpha$ is continuous, because it's composed by a homeomorphism and a path. It remains to show, that for all $k \in 1, \ldots n$ :

$$
\begin{aligned}
& \alpha\left(\frac{k}{n}\right)=\lim _{\tau \searrow \frac{k}{n}} \alpha(\tau)=\phi_{x_{k}}^{-1}\left(\beta\left(\frac{k}{n}\right), \operatorname{pr}_{\pi^{-1}\left\{x_{k}\right\}} \circ \phi_{x_{k}}\left(\alpha\left(\frac{k}{n}\right)\right)\right) \Longleftrightarrow \\
\Longleftrightarrow & \phi_{x_{k}}\left(\alpha\left(\frac{k}{n}\right)\right)=\left(\beta\left(\frac{k}{n}\right), \operatorname{pr}_{\pi^{-1}\left\{x_{k}\right\}} \circ \phi_{x_{k}}\left(\alpha\left(\frac{k}{n}\right)\right)\right) \Longleftrightarrow \operatorname{pr}_{U_{x_{k}}} \circ \phi_{x_{k}}\left(\alpha\left(\frac{k}{n}\right)\right)=\beta\left(\frac{k}{n}\right) \Longleftrightarrow \\
\Longleftrightarrow & \pi\left(\alpha\left(\frac{k}{n}\right)\right)=\beta\left(\frac{k}{n}\right) \text { and that's true given (c). }
\end{aligned}
$$

After all, we constructed a path $\alpha:[0,1] \rightarrow B$, which has the start point $\alpha(0)=w$ and is a lift of $\beta$ over $\pi$ : $\pi \circ \alpha=\beta$.

2.8. Corollary (unwinding). Each curve on a generalized cylinder can be lifted onto a real vector space:

Let be $\xi:[0, \lambda] \rightarrow \mathbb{R}^{2}$ the simple closed path, which is parameterized by arc length and generates the cylinder:

$$
\mathcal{Z}=\operatorname{im}(\xi) \times \mathbb{R}^{m} \subset \mathbb{R}^{2} \times \mathbb{R}^{m}
$$

Its continuous continuation $\hat{\xi}$ produces the cover (see lemma 2.5):

$$
\pi:=\hat{\xi} \times \mathrm{id}: \mathbb{R} \times \mathbb{R}^{m} \rightarrow \mathcal{Z}
$$

Given theorem 2.7, each path $\beta:[0, a L] \rightarrow \mathcal{Z}$ has a unique lift $\alpha:[0, a L] \rightarrow \mathbb{R} \times \mathbb{R}^{m}$ such that:

$$
\pi \circ \alpha=\beta \text { and } \alpha(0)=\left.\pi\right|_{\left[0, \lambda\left[\times \mathbb{R}^{m}\right.\right.} ^{-1}(\beta(0))
$$

Proof. It only remains to show, that $\pi$ restricted to $\left[0, \lambda\left[\times \mathbb{R}^{m}\right.\right.$ is bijective. But since $\xi$ is simple closed and hence injective on $[0, \lambda[$, the following restriction is bijective:

$$
\left.\pi\right|_{\left[0, \lambda\left[\times \mathbb{R}^{m}\right.\right.}=\left.\xi\right|_{[0, \lambda[} \times \mathrm{id}:\left[0, \lambda\left[\times \mathbb{R}^{m} \rightarrow \operatorname{im}(\xi) \times \mathbb{R}^{m}\right.\right.
$$




\subsection{Convergence}

2.9. Definition (geodesic). The cylinder $\mathcal{Z} \subset\left(\mathbb{R}^{2} \times \mathbb{R}^{m},\|\cdot\|_{2}\right)$ is metrizable. For points $x, y \in \mathcal{Z}$ we define:

$$
d(x, y):=\inf \left\{\ell(\beta):=\sup _{r=t_{0}<\cdots<t_{k}=s} \sum_{j=1}^{k}\left\|\beta\left(t_{j}\right)-\beta\left(t_{j-1}\right)\right\|_{2} \mid \beta:[r, s] \rightarrow \mathcal{Z}, \beta(r)=x, \beta(s)=y\right\}
$$

A geodesic $\psi:[0,1] \rightarrow \mathcal{Z}$ is a locally shortest path, i.e., there's a constant velocity $v \geq 0$ such that:

$$
\forall t \in[0,1]: \exists \delta>0: \forall r, s \in] t-\delta, t+\delta[: d(\psi(r), \psi(s))=v|r-s|
$$
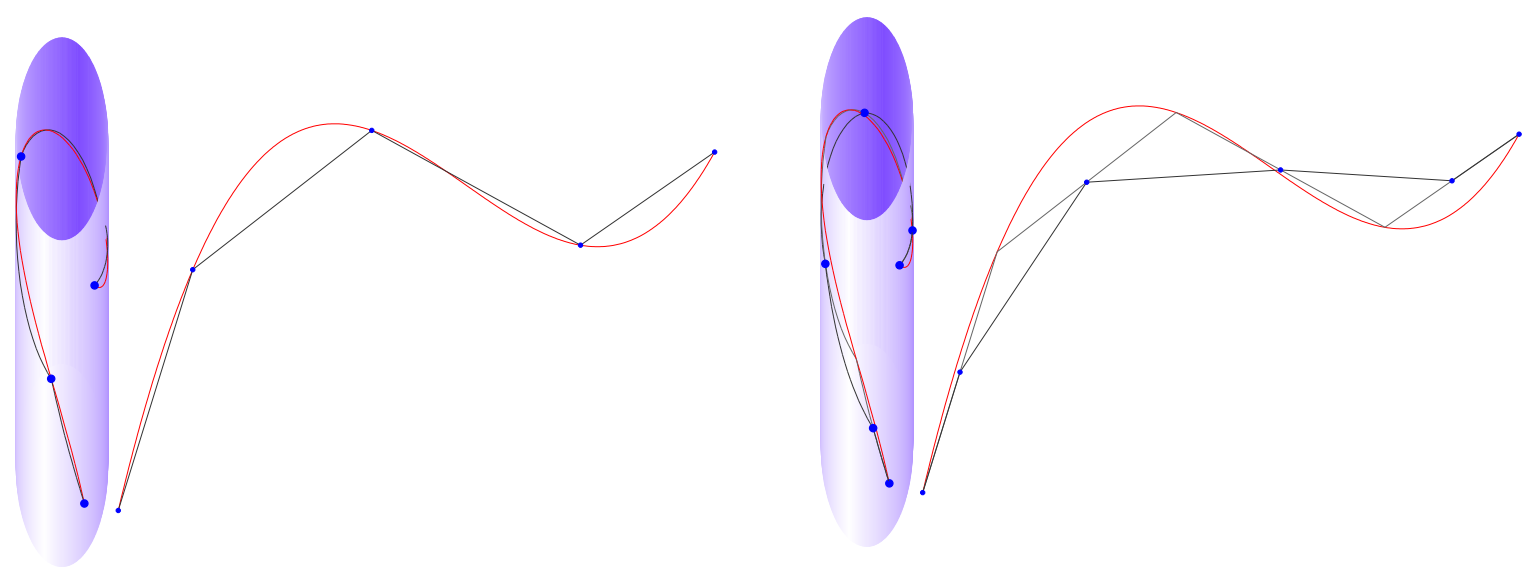

2.10. Remark (short ruler method). Let $\beta:[0, a L] \rightarrow \mathcal{Z}=\operatorname{im}(\xi) \times \mathbb{R}^{m}$ be a path on a generalized cylinder parameterized by arc length and $q_{k}:=\beta(k L)$ the constructed nodes of a polygonal geodesic.

The map $\pi:=\hat{\xi} \times \mathrm{id}: \mathbb{R} \times \mathbb{R}^{m} \rightarrow \mathcal{Z}$ covers the cylinder, for an arbitrary start vector $w \in \pi^{-1}(\beta(0))$ we get:

(1) Lifting $\beta$ by the cover $\pi$ leads to a unique path with start point $w$ in the vector space:

$$
\alpha:[0, a L] \rightarrow \mathbb{R} \times \mathbb{R}^{m} \text { with } \beta=\pi \circ \alpha \text { and } \alpha(0)=w
$$

(2) The constructed nodes $p_{k} \in \mathbb{R} \times \mathbb{R}^{m}$ from $\alpha$ correspond to the constructed nodes from $\beta$ on the cylinder:

$$
q_{k}=\beta(k L)=\pi \circ \alpha(k L)=\pi\left(p_{k}\right)
$$

(3) Applying repeatedly the midpoint method to the points $p$ in the vector space $\mathbb{R} \times \mathbb{R}^{m}$ generates nodes of polygonal geodesics on the cylinder:

$$
q_{k}^{t}:=\pi\left(\left(S^{t} p\right)_{k}\right) \in \mathcal{Z} \text { for } k \in \mathbb{Z}
$$



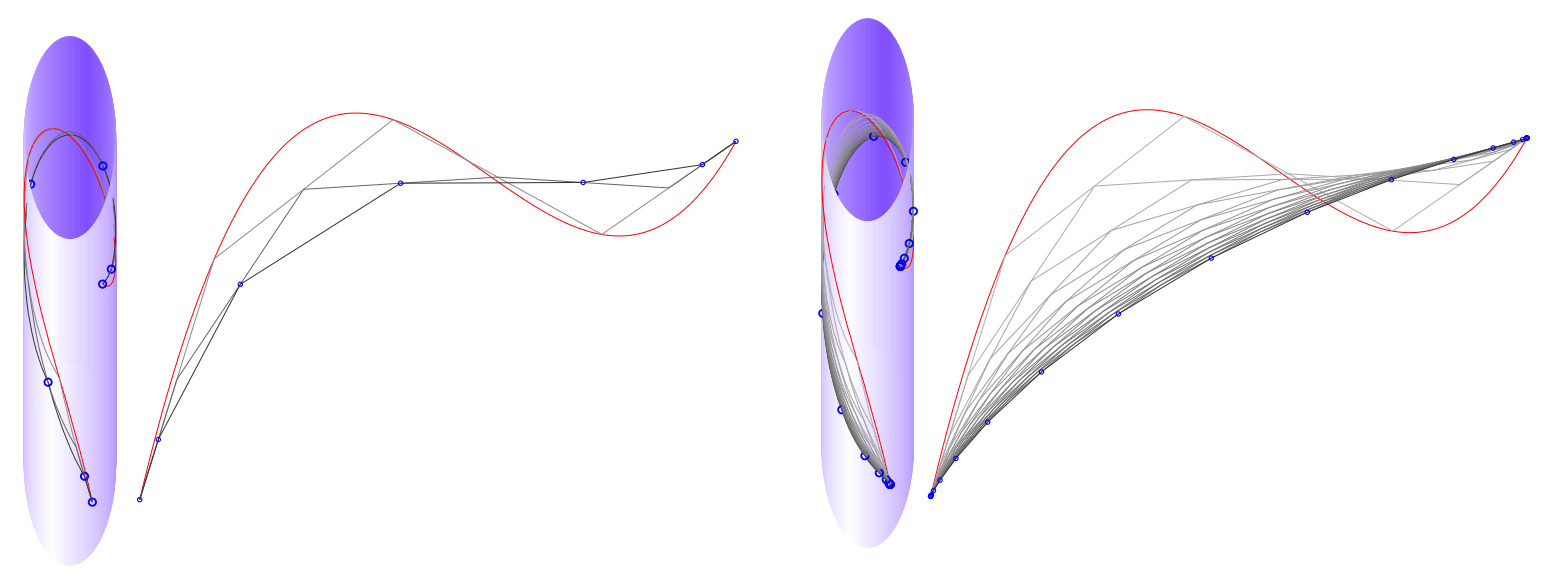

2.11. Lemma. The map $\pi=\hat{\xi} \times \operatorname{id}_{\mathbb{R}^{m}}$ covers the generalized cylinder $\mathcal{Z}=\operatorname{im}(\xi) \times \mathbb{R}^{m}$ given lemma 2.5. Let the path $\varphi:[0,1] \rightarrow \mathbb{R} \times \mathbb{R}^{m}$ have constant derivative $\dot{\varphi}$, i.e., it parameterizes a straight line by constant velocity.

Then, the cover transports it to the geodesic $\psi:=\pi \circ \varphi$ on the cylinder $(\mathcal{Z}, d)$.

Proof. (a) The straight line $\varphi(t)=\varphi(0)+t \dot{\varphi}$ parameterizes a straight line by constant velocity $v:=\|\dot{\varphi}\|_{2}$. Since the cover $\pi: \mathbb{R} \times \mathbb{R}^{m} \rightarrow \mathcal{Z}$ is continuous, it transports $\varphi$ to the path $\psi=\pi \circ \varphi$ on the cylinder.

(b) All paths in this proof are either in $\mathbb{R} \times \mathbb{R}^{m}$ or in $\mathbb{R}^{2} \times \mathbb{R}^{m}$. Each vector $u$ in this spaces can be decomposed: $u=\left(u_{x}, u_{y}\right)$. Hereby, $u_{x}$ denotes the first (one or two) and $u_{y}$ the last $m$ components.

Then, it holds that:

$$
\|u\|_{2}=\sqrt{\sum_{j}\left|u_{j}\right|^{2}}=\sqrt{\left\|u_{x}\right\|_{2}^{2}+\left\|u_{y}\right\|_{2}^{2}}
$$

(c) For all $r, s \in[0,1]$ the distance between the points $\psi(r)$ and $\psi(s)$ on the cylinder is at most $v|r-s|$, since the distance is the infimum of all lengths of paths, which connect the two points on the cylinder, and $\left.\psi\right|_{[r, s]}$ is such a path. Because without loss of generality, we assume $r<s$ and it holds that:

$$
\begin{aligned}
d(\psi(r), \psi(s)) & \leq \ell\left(\left.\psi\right|_{[r, s]}\right)=\sup _{r=t_{0}<\cdots<t_{k}=s} \sum_{j=1}^{k}\left\|\psi\left(t_{j}\right)-\psi\left(t_{j-1}\right)\right\|_{2} \stackrel{(b)}{=} \\
& =\sup _{r=t_{0}<\cdots<t_{k}=s} \sum_{j=1}^{k} \sqrt{\left\|\psi\left(t_{j}\right)_{x}-\psi\left(t_{j-1}\right)_{x}\right\|_{2}^{2}+\left\|\psi\left(t_{j}\right)_{y}-\psi\left(t_{j-1}\right)_{y}\right\|_{2}^{2}}
\end{aligned}
$$

Given (a), the path $\psi$ is $\pi \circ \varphi=(\hat{\xi} \times$ id $) \circ \varphi$ : The first components are $\psi(t)_{x}=\hat{\xi}\left(\varphi(t)_{x}\right)$ and the last components are $\psi(t)_{y}=\varphi(t)_{y}$. Since $\hat{\xi}$ is parameterized by arc length, it holds that:

$$
\left\|\psi\left(t_{j}\right)_{x}-\psi\left(t_{j-1}\right)_{x}\right\|_{2}=\left\|\hat{\xi}\left(\varphi\left(t_{j}\right)_{x}\right)-\hat{\xi}\left(\varphi\left(t_{j-1}\right)_{x}\right)\right\|_{2} \leq \ell\left(\left.\hat{\xi}\right|_{\left[\varphi\left(t_{j-1}\right)_{x}, \varphi\left(t_{j}\right)_{x}\right]}\right)=\left|\varphi\left(t_{j}\right)_{x}-\varphi\left(t_{j-1}\right)_{x}\right|
$$


Together with the parameterization $\varphi(t)=\varphi(0)+t \dot{\varphi}$ from (a) the distance is at most:

$$
\begin{aligned}
d(\psi(r), \psi(s)) & \leq \sup _{r=t_{0}<\cdots<t_{k}=s} \sum_{j=1}^{k} \sqrt{\left|\varphi\left(t_{j}\right)_{x}-\varphi\left(t_{j-1}\right)_{x}\right|^{2}+\left\|\varphi\left(t_{j}\right)_{y}-\varphi\left(t_{j-1}\right)_{y}\right\|_{2}^{2}} \stackrel{(b)}{=} \\
& =\sup _{r=t_{0}<\cdots<t_{k}=s} \sum_{j=1}^{k}\left\|\varphi\left(t_{j}\right)-\varphi\left(t_{j-1}\right)\right\|_{2} \stackrel{(a)}{=} \sup _{r=t_{0}<\cdots<t_{k}=s} \sum_{j=1}^{k}\left\|\varphi(\theta)+t_{j} \dot{\varphi}-\varphi(\theta)-t_{j-1} \dot{\varphi}\right\|_{2}= \\
& =\sup _{r=t_{0}<\cdots<t_{k}=s} \sum_{j=1}^{k}\left|t_{j}-t_{j-1}\right| \underbrace{\|\dot{\varphi}\|_{2}}_{\stackrel{(a)}{=} v}=|r-s| v
\end{aligned}
$$

(d) It remains to show, that on the other side for all $t \in[0,1]$ there exists a $\delta>0$, such that for all $r$ and $s$ in the neighborhood $] t-\delta, t+\delta[$ of $t$ the distance $d(\psi(r), \psi(s))$ is at least $v|r-s|$.

We choose $\delta:=\lambda / 4 v$ for all $t$ and $r, s \in] t-\delta, t+\delta$ [ arbitrarily. Without loss of generality, we assume that $r$ is less than $s$. The distance between $\psi(r)$ and $\psi(s)$ is the infimum of all lengths of paths, which connect the two points on the cylinder. Let be $\beta:[r, s] \rightarrow \mathcal{Z}$ such a path.

Given theorem 2.7 and since $\pi \circ \varphi(r)=\psi(r)$, there's an unique path:

$$
\alpha:[r, s] \rightarrow \mathbb{R} \times \mathbb{R}^{m} \text { with }(\hat{\xi} \times \text { id }) \circ \alpha=\beta \text { and } \alpha(r)=\varphi(r)
$$

$1^{\text {st }}$ case: There's a $\sigma \in[r, s]$ with $\alpha(\sigma)_{x} \in\left\{\alpha(r)_{x} \pm \lambda / 2\right\}$. Then, the path $\beta$ has at least the length:

$$
\begin{aligned}
\ell(\beta) & \geq \ell\left(\left.\beta\right|_{[r, \sigma]}\right)=\sup _{r=\tau_{0}<\cdots<\tau_{k}=\sigma} \sum_{j=1}^{k}\left\|\beta\left(\tau_{j}\right)-\beta\left(\tau_{j-1}\right)\right\|_{2} \geq \sup _{r=\tau_{0}<\cdots<\tau_{k}=\sigma} \sum_{j=1}^{k}\left\|\beta\left(\tau_{j}\right)_{x}-\beta\left(\tau_{j-1}\right)_{x}\right\|_{2} \stackrel{(d)}{=} \\
& =\sup _{r=\tau_{0}<\cdots<\tau_{k}=\sigma} \sum_{j=1}^{k}\left\|\hat{\xi}\left(\alpha\left(\tau_{j}\right)_{x}\right)-\hat{\xi}\left(\alpha\left(\tau_{j-1}\right)_{x}\right)\right\|_{2}=\sup _{r=\tau_{0}<\cdots<\tau_{k}=\sigma} \sum_{j=1}^{k} \ell\left(\left.\hat{\xi}\right|_{\left[\alpha\left(\tau_{j-1}\right)_{x}, \alpha\left(\tau_{j}\right)_{x}\right]}\right) \geq \\
& \geq \ell\left(\left.\hat{\xi}\right|_{\left[\alpha(r)_{x}, \alpha(\sigma)_{x}\right]}\right)={ }_{\hat{\xi} \text { parameterized by arc length }}\left|\alpha(r)_{x}-\alpha(\sigma)_{x}\right|=\left|\alpha(r)_{x}-\alpha(r)_{x} \pm \frac{\lambda}{2}\right|=2 \delta v
\end{aligned}
$$

Since $r$ and $s$ are in the $\delta$-neighborhood of $t$, the distance $|r-s|$ is less than $2 \delta$ and altogether it holds that:

$$
\ell(\beta) \geq 2 \delta v>|r-s| v
$$

$2^{\text {nd }}$ case: For each $\sigma \in[r, s]$ it's $\alpha(\sigma)_{x} \notin\left\{\alpha(r)_{x} \pm \lambda / 2\right\}$. Then, the path $\alpha$ remains in the open neighborhood:

$$
V:=] \alpha(r)_{x}-\frac{\lambda}{2}, \alpha(r)_{x}+\frac{\lambda}{2}\left[\times \mathbb{R}^{m}=\alpha(r)+\right]-\frac{\lambda}{2}, \frac{\lambda}{2}\left[\times \mathbb{R}^{m} \supset \operatorname{im}(\alpha)\right.
$$

Like $\alpha$, the path $\varphi$ remains in $V$ : Given the parameterization $\varphi(t)=\varphi(0)+t \dot{\varphi}$ from (a), for all $\sigma \in[r, s]$ it's:

$$
\begin{aligned}
& \varphi(\sigma) \stackrel{(a)}{=} \varphi(0)+\dot{\varphi} \sigma \stackrel{(a)}{=} \overbrace{\varphi(r)}^{=\alpha(r)}+\dot{\varphi}(\sigma-r) \stackrel{r \leq \sigma \leq s}{\in} \alpha(r)+\dot{\varphi}[0, \overbrace{s-r}^{<2 \delta}] \stackrel{\delta=\frac{\lambda}{4 v}}{\subseteq} \\
& \subseteq \alpha(r)+\dot{\varphi}\left[0, \frac{\lambda}{2 v}[\stackrel{v=\|\dot{\varphi}\|}{=} \alpha(r)+\overbrace{\frac{\dot{\varphi}}{\|\dot{\varphi}\|}}^{\in \overline{B_{1}(0)}}\left[0, \frac{\lambda}{2}[\subseteq \alpha(r)+]-\frac{\lambda}{2}, \frac{\lambda}{2}\left[\times \mathbb{R}^{m}=V\right.\right.\right.
\end{aligned}
$$


Thus, the restriction $\left.\pi\right|_{V}$ transports the paths $\alpha$ and $\varphi$ to $\beta$ and $\psi$, respectively, and $\alpha(s)$ equals $\varphi(s)$, because $\pi_{V}$ is homeomorphic like in the proof to lemma 2.5 and:

$$
\left.\pi\right|_{V}(\alpha(s))=\beta(s)=\psi(s)=\left.\pi\right|_{V}(\varphi(s))
$$

Given this and the parameterization $\varphi(\tau)=\varphi(0)+\tau \dot{\varphi}$ from (a), it holds that:

$$
\|\alpha(r)-\alpha(s)\|_{2}=\|\varphi(r)-\varphi(s)\|_{2}=\|\varphi(\theta)+r \dot{\varphi}-\varphi(\theta)-s \dot{\varphi}\|_{2}=|r-s|\|\dot{\varphi}\|_{2}=v|r-s|
$$

Since the length of $\beta_{x}$ is the supremum of lengths of approximating polygonal lines, for an arbitrary $\varepsilon>0$ there's a dissection $r=\sigma_{0}<\cdots<\sigma_{l}=s$ with:

$$
\varepsilon+\sum_{j=0}^{l}\left\|\beta\left(\sigma_{j}\right)_{x}-\beta\left(\sigma_{j-1}\right)_{x}\right\|_{2} \geq \sup _{r=\tau_{0}<\cdots<\tau_{k}=s} \sum_{j=1}^{k}\left\|\beta\left(\tau_{j}\right)_{x}-\beta\left(\tau_{j-1}\right)_{x}\right\|_{2}=\ell\left(\beta_{x}\right)=\sum_{j=0}^{l} \ell\left(\left.\beta_{x}\right|_{\left[\sigma_{j-1}, \sigma_{j}\right]}\right)
$$

There are $\left(\varepsilon_{j} \geq 0\right)_{j=1}^{l}$ with $\sum_{j=1}^{l} \varepsilon_{j}=\varepsilon$ such that $\varepsilon_{j}+\left\|\beta\left(\sigma_{j}\right)_{x}-\beta\left(\sigma_{j-1}\right)_{x}\right\|_{2} \geq \ell\left(\left.\beta_{x}\right|_{\left[\sigma_{j-1}, \sigma_{j}\right]}\right)=\ell\left(\left.\hat{\xi} \circ \alpha_{x}\right|_{\left[\sigma_{j-1}, \sigma_{j}\right]}\right)$ and it holds that:

$$
\begin{aligned}
& \varepsilon+\ell(\beta) \geq \sum_{j=1}^{l} \varepsilon_{j}+\left\|\beta\left(\sigma_{j}\right)-\beta\left(\sigma_{j-1}\right)\right\|_{2} \stackrel{(b)}{=} \sum_{j=1}^{l} \varepsilon_{j}+\sqrt{\left\|\beta\left(\sigma_{j}\right)_{x}-\beta\left(\sigma_{j-1}\right)_{x}\right\|_{2}^{2}+\left\|\beta\left(\sigma_{j}\right)_{y}-\beta\left(\sigma_{j-1}\right)_{y}\right\|_{2}^{2}} \geq_{\text {equality }}^{\text {triangle }} \\
& \geq \sum_{j=1}^{l} \sqrt{\left(\varepsilon_{j}+\left\|\beta\left(\sigma_{j}\right)_{x}-\beta\left(\sigma_{j-1}\right)_{x}\right\|_{2}\right)^{2}+\left\|\beta\left(\sigma_{j}\right)_{y}-\beta\left(\sigma_{j-1}\right)_{y}\right\|_{2}^{2}} \geq\left[\begin{array}{l}
\varepsilon_{j}+\left\|\beta\left(\sigma_{j}\right)_{x}-\beta\left(\sigma_{j-1}\right)_{x}\right\|_{2} \geq \ell\left(\left.\hat{\xi} \circ \alpha_{x}\right|_{\left[\sigma_{j-1}, \sigma_{j}\right]}\right) \geq \\
\geq \ell\left(\left.\hat{\xi}\right|_{\left[\alpha\left(\sigma_{j-1}\right)_{x}, \alpha\left(\sigma_{j}\right)_{x}\right]}\right) \stackrel{\text { p.a.l. }}{=}\left|\alpha\left(\sigma_{j}\right)_{x}-\alpha\left(\sigma_{j-1}\right)_{x}\right|
\end{array}\right] \\
& \geq \sum_{j=1}^{l} \sqrt{\left|\alpha\left(\sigma_{j}\right)_{x}-\alpha\left(\sigma_{j-1}\right)_{x}\right|^{2}+\left\|\beta\left(\sigma_{j}\right)_{y}-\beta\left(\sigma_{j-1}\right)_{y}\right\|_{2}^{2}}=\sum_{j=1}^{l} \sqrt{\left|\alpha\left(\sigma_{j}\right)_{x}-\alpha\left(\sigma_{j-1}\right)_{x}\right|^{2}+\left\|\alpha\left(\sigma_{j}\right)_{y}-\alpha\left(\sigma_{j-1}\right)_{y}\right\|_{2}^{2}} \\
& \stackrel{(b)}{=} \sum_{j=1}^{l}\left\|\alpha\left(\sigma_{j}\right)-\alpha\left(\sigma_{j-1}\right)\right\|_{2} \geq_{\text {equality }}^{\text {triangle }}\|\alpha(s)-\alpha(r)\|_{2} \stackrel{(1)}{=} v|r-s|
\end{aligned}
$$

That's true for arbitrary $\epsilon>0$ and hence is $\ell(\beta) \geq v|r-s|$, like in the first case.

(f) All paths $\beta:[r, s] \rightarrow \mathbb{Z}$ connecting the points $\psi(r)$ and $\psi(s)$ have length $\ell(\beta) \geq v|r-s|$. Thus, the distance between the two points is as infimum of lengths of such paths also at least $v|r-s|$.

Altogether, for $r, s \in] t-\delta, t+\delta[$ the distance $d(\psi(r), \psi(s))$ is on one side at most $v|r-s|$, on the other side at least $v|r-s|$ and so it must be equal. 


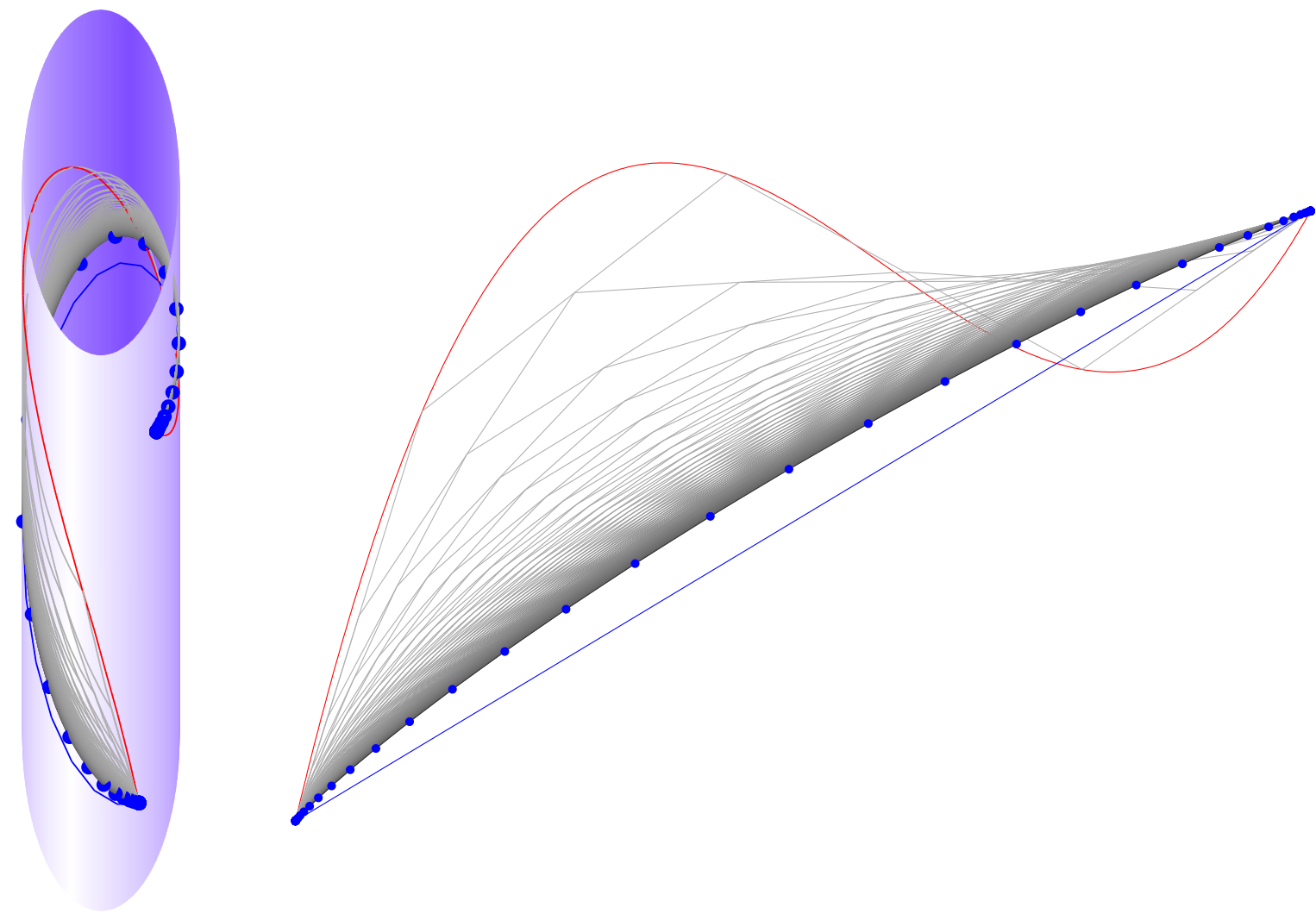

2.12. Theorem (convergence). The generated polygonal geodesics converge to the following path between start and end point of $\beta$ :

$$
\gamma:[0,1] \longrightarrow \mathcal{Z}, \tau \longmapsto \pi(\alpha(0)+\tau(\alpha(a L)-\alpha(0)))
$$

This geodesic is homotopic to $\beta$ but in general not a shortest path between start and end point.

Proof. The nodes $p_{k}^{t}=\left(S^{t} p\right)_{k}$ generate a polygonal line in the vector space: $\varphi^{t}:[0,1] \rightarrow \mathbb{R} \times \mathbb{R}^{m}$. Transporting it onto the cylinder generates the polygonal geodesic $\psi^{t}:=\pi \circ \varphi^{t}$ with nodes $q_{k}^{t}=\pi\left(p_{k}^{t}\right)$.

Given theorem 1.5 the polygonal lines $\varphi^{t}$ converge in the vector space $\mathbb{R} \times \mathbb{R}^{m}$ to the straight line between $\alpha(0)=p_{0}$ and $\alpha(a L)=p_{n}$.

Let the polygonal lines $\varphi^{t}$ be parameterized WLOG so, that for all $\tau \in[0,1]$ the points $\varphi^{t}(\tau)$ converge to $\alpha(0)+\tau(\alpha(a L)-\alpha(0))=: \chi(\tau)$. Since $\pi$ is continuous for all $\tau \in[0,1]$, it holds that:

$$
\lim _{t \rightarrow \infty} \psi^{t}(\tau)=\lim _{t \rightarrow \infty} \pi\left(\varphi^{t}(\tau)\right)=\pi\left(\lim _{t \rightarrow \infty} \varphi^{t}(\tau)\right)=\pi(\alpha(0)+\tau(\alpha(a L)-\alpha(0)))=\gamma(\tau)
$$

(a) Given lemma 2.11, the cover $\pi$ transports the straight line $\chi$ from the vector space $\mathbb{R} \times \mathbb{R}^{m}$ to the geodesic $\gamma=\pi \circ \chi$ on the cylinder.

(b) In the normed vector space $\left(\mathbb{R} \times \mathbb{R}^{m},\|\cdot\|\right)$ there's clearly a homotopy between the paths $\alpha$ and $\chi$. The cover $\pi$ is continuous and transports it onto the cylinder. Hence, the paths $\beta=\pi \circ \alpha$ and $\gamma=\pi \circ \chi$ are homotopic.

(c) The short ruler method doesn't yield always a shortest path, e.g. start and end point of the path $\beta:[0, \lambda] \rightarrow \mathcal{Z}: \tau \mapsto(\xi(\tau), 0)$ coincide, because $\xi$ is simple closed: $\beta(0)=(\xi(0), 0)=(\xi(\lambda), 0)=\beta(\lambda)$. It's a geodesic and a fixed point of the midpoint method. Thus, $\gamma$ is a reparameterization of $\beta$ and has the length:

$$
\ell(\gamma)=\ell(\beta)=\ell(\xi)=\lambda>0
$$


But the shortest curve has the length 0 as it's constant: $\tau \mapsto(\xi(0), 0)$.

Altogether, the polygonal lines of the short ruler method converge to the geodesic $\gamma$, which is homotopic to $\beta$ but in general not a shortest path.

\section{REFERENCES}

[1] Gerd Laures and Markus Szymik. Grundkurs Topologie. Spektrum Akademischer Verlag, Heidelberg, 2000.

[2] Tammo tom Dieck. Topologie. de Gruyter, Berlin, 1991.

[3] Otto Forster. Lectures on Riemann surfaces. Springer, Corr. 2. print. New York, 1991. 